\title{
Interpenetrating polyaniline-gold electrodes for SERS and electrochemical measurements
}

\author{
R. M. West ${ }^{a^{*}}$ and S. Semancik ${ }^{b}$ \\ ${ }^{a}$ Current address: The University of San Francisco, Chemistry Department, 2130 Fulton St, San Francisco, CA 94117 \\ ${ }^{b}$ National Institute of Standards and Technology, 100 Bureau Dr, MS 8362, Gaithersburg, MD 20899 \\ *Corresponding author e-mail: rmwest2@usfca.edu
}

Facile fabrication of nanostructured electrode arrays is critical for development of bimodal SERS and electrochemical biosensors. In this paper, the variation of applied potential at a polyaniline-coated Pt electrode is used to selectivity deposit Au on the polyaniline amine sites or on the underlying Pt electrode. By alternating the applied potential, the Au is grown simultaneously from the top and the bottom of the polyaniline film, leading to an interpenetrated, nanostructured polymer-metal composite extending from the Pt electrode to the electrolyte solution. The resulting films have unique $\mathrm{pH}$-dependent electrochemical properties, e.g. they retain electrochemical activity in both acidic and neutral solutions, and they are composed of SERS-active nanostructures. By varying the concentration of chloroaurate used during deposition, Au nanoparticles, nanodendrites, or nanosheets can be selectively grown. For the films deposited under optimal conditions, using $5 \mathrm{mmol} / \mathrm{L}$ chloroaurate, the SERS enhancement factor for rhodamine $6 \mathrm{G}$ was found to be as high as $1.1 \times 10^{6}$ with spot-to-spot and electrode-to-electrode relative standard deviations as low as $8 \%$ and $12 \%$, respectively. The advantages of the reported PANI-Au composite electrodes lie in their facile fabrication, enabling the targeted deposition of tunable nanostructures on sensing arrays, and their ability to produce orthogonal optical and electrochemical analytical results.

\section{Keywords:}

Electrodeposition; Polyaniline; Nanostructured Gold; SERS; Bimodal; Biosensing

\section{Introduction}

Simultaneous bimodal electrochemical (EC) and surface enhanced Raman spectroscopy (SERS) measurements can enhance a sensor's figures-of-merit, e.g. limit of detection, sensitivity, dynamic range, selectivity and response time; thus improving its reliability in critical applications. [1] These techniques have already been combined to form a powerful spectroelectrochemical tool that can monitor chemical reactions at surfaces, probe interfacial interactions, and characterize molecular orientations in real-time, but utilization towards biologically relevant examples is rare.[2-5] A key factor for EC-SERS measurements is the fabrication of stable, SERS-active electrodes with a uniform and reproducible enhancement factor, EF. Furthermore, facile and inexpensive fabrication of the electrodes in an array format is desirable for parallel, multiplexed measurements.

SERS provides molecule-specific vibrational signatures like standard Raman spectroscopy but with signal enhancements typically $10^{4}$ to $10^{8}$. This amplification is localized to the surface of the SERS substrate and is attributed to two mechanisms: electromagnetic enhancement and chemical enhancement.[6] The former offers the larger enhancement $\left(10^{4}\right.$ to $\left.10^{7}\right)$ and is the result of localized surface plasmons.[7] In particular, the regions between closely spaced nanoparticles provide hot-spots with dramatically increased enhancement. The chemical enhancement, on the other hand, is the outcome of charge-transfer between the analyte and the surface and contributes only a factor of 10 to $10^{2}$ of the total enhancement. Besides the SERS performance, another important issue to consider for EC-SERS applications is the electrochemical performance of the 
SERS-active surface. In this regard, Au possesses superior electrochemical stability compared to Ag. Furthermore, if properly designed, enhancement factors of Au substrates are comparable to those of Ag substrates.[7] Because SERS provides molecule-specific vibrational signatures and has the potential for very low detection limits, even single molecule detection, [8] it holds much promise for chemical and biological sensing applications.[9] When coupled to simultaneous electrochemical modulation and/or sensing, the resulting bimodal technique can provide new insight into biochemical processes at electrode surfaces, leading to new sensing possibilities.

Recently, there has been much interest in conducting polymer-metallic nanoparticle composites because of their unique physical, electrical and optical properties, as well as their potential applications in biochemical sensing, organic electronics, nonvolatile memory, and catalysis.[10-17] Of particular interest are polyaniline-gold (PANI-Au) nanocomposites,[11, 18] in part because polyaniline provides a unique template for the binding and reduction of chloroaurate at its nitrogen linkages. The unique properties of PANI-Au composites are welladapted for next generation sensing. [19,20] Controlling the film morphology and gold nanostructure has been addressed through various routes, including spontaneous chloroaurate reduction by PANI,[21, 22] in-situ aniline polymerization and simultaneous chloroaurate reduction, [23, 24] binding of pre-synthesized gold nanoparticles on polyaniline films, [21] electrochemically controlled growth,[25] seeded growth,[26] and electrochemical dissolution of gold electrodes underneath PANI films.[27] While there have been some advances towards SERS-active PANI-Au materials, so far the resulting films are typically composed of sparse gold nanoparticles embedded in PANI. Furthermore, PANI-Au films that can be used as electrodes at neutral $\mathrm{pH}$, where polyaniline has low conductivity, have not been developed.[21]'[22]'[28]

In this paper, we present a facile electrochemical procedure for the fabrication of SERS-active and electrochemically-active PANI-Au electrodes. Using a method adapted from one reported by Janata et al.,[29] we utilize the unique redox properties of PANI-coated electrodes to laterally shift the electrochemical interface during Au deposition, thus avoiding the formation of isolated gold nanoparticles while still retaining the nanostructures necessary for SERS. By alternating the location of Au deposition between the PANI film and the underlying Pt electrode, we employ PANI as a template for the growth of a dense, nanostructured, and electrochemically-active electrode. In this method, PANI not only provides the binding sites for chloroaurate at its nitrogen linkages, but also allows electrochemical control of where the chloroaurate is reduced, in the film or at the underlying Pt electrode. This control is key for the morphology of the resulting gold nanostructures, and it enables electrochemical activity of the interpenetrating PANI-Au electrodes at neutral $\mathrm{pH}$, a critical attribute for biosensing applications. Additionally, this method is realized completely via electrochemical processing in under 30 minutes, facilitating targeted deposition of tuned plasmonic nanostructures on individually addressable electrode arrays for multiplexed, orthogonal sensing applications.

\section{Experimental}

[Certain commercial equipment, instruments, or materials are identified in this document. Such identification is not intended to imply recommendation or endorsement by the National Institute of Standards and Technology, nor is it intended to imply that the products identified are necessarily the best available for the purpose.]

\subsection{Chemicals}

Tetrafluoroboric acid (Sigma-Aldrich, 48 wt \% in $\mathrm{H}_{2} \mathrm{O}$ ), aniline (Sigma-Aldrich, $\geq 99.5$ wt \%), potassium gold chloride (Aldrich, 99.995 wt \%), rhodamine $6 \mathrm{G}$ chloride (Exciton, Inc), sulfuric acid (J.T. Baker), hydrogen peroxide (Sigma-Aldrich, $30 \mathrm{wt} \%$ in $\mathrm{H}_{2} \mathrm{O}$ ), potassium chloride (J.T. Baker), 4-mercaptobenzoic acid (Sigma-Aldrich, $99 \mathrm{wt} \%$ ) and potassium ferricyanide (III) (Aldrich, > 99 wt \%) were all used as received. 


\subsection{Electrode Array Fabrication}

The 16-channel electrode array used in all reported studies was fabricated in the NIST Center for Nanoscale Science and Technology (CNST) NanoFab cleanroom. This array allowed efficient examination of deposition parameters. Standard photolithographic techniques were used to develop the array pattern on a 4-inch $\mathrm{Si}$ wafer with $200 \mathrm{~nm}$ thermally grown $\mathrm{SiO}_{2}$ (WRS Wafers). A $10 \mathrm{~nm}$ titanium adhesion layer and $200 \mathrm{~nm}$ platinum layer were deposited using E-beam evaporation and patterned into electrodes, leads, and bonding pads using standard lift-off processing. An $800 \mathrm{~nm} \mathrm{SiO}_{2}$ passivation layer was deposited using plasma enhanced chemical vapor deposition. The openings in the $\mathrm{SiO}_{2}$ for the bonding pads and electrodes were etched using Buffer Oxide Etch.

The electrodes are located at one end of a $50 \mathrm{~mm}$ by $12 \mathrm{~mm} \mathrm{SiO}{ }_{2}$ "dipstick" that is diced from the wafer. The other end of this dipstick is epoxied and wire-bonded to a PC board with card-edge reader (Sunstone Circuits) for easy connection to one or more of the electrodes simultaneously. Each Pt working electrode has a diameter of $1 \mathrm{~mm}$. Before use, the individually addressable electrodes are cleaned in a two-step process: (1) immersion in piranha solution (3 to 1 concentrated sulfuric acid to $30 \%$ hydrogen peroxide) for 1 minute to remove residual photoresist, and (2) electrochemical polishing by consecutive pulsing and cycling in $0.1 \mathrm{~mol} / \mathrm{L} \mathrm{H}_{2} \mathrm{SO}_{4}$. After cleaning, each electrode is cycled in $2.5 \mathrm{mmol} / \mathrm{L}$ potassium ferricyanide in $0.1 \mathrm{~mol} / \mathrm{L} \mathrm{KCl}$ to confirm electroactivity.

\subsection{Electrochemical Measurements}

All electrochemical measurements and depositions were carried out with a Solartron SI 1287 Electrochemical Workstation (Solartron Analytical) controlled by CorrWare software (Scribner Associates, Inc). The working electrodes were Pt disc electrodes (see above). The reference electrode was a single junction $\mathrm{Ag} / \mathrm{AgCl}$ (in $1 \mathrm{~mol} / \mathrm{L} \mathrm{KCl}$ ). A Pt wire was used as a counter electrode. During electrochemical characterizations, cyclic voltammograms (CVs) were carried out in $2 \mathrm{~mol} / \mathrm{L} \mathrm{HBF}_{4}$ from $-0.2 \mathrm{~V}$ to $0.8 \mathrm{~V}$ or in $2.5 \mathrm{mmol} / \mathrm{L}$ potassium ferricyanide in $0.1 \mathrm{M} \mathrm{KCl}$ from $0.0 \mathrm{~V}$ to $0.4 \mathrm{~V}$, starting at open cell potential and cycled until stable (typically no more than 5 cycles) at a scan rate of $50 \mathrm{mV} / \mathrm{s}$.

\subsection{Electrodeposition of Polyaniline}

Polyaniline was electropolymerized from $0.1 \mathrm{~mol} / \mathrm{L}$ aniline in $2 \mathrm{~mol} / \mathrm{L} \mathrm{HBF}_{4}$ by applying a constant potential of $0.9 \mathrm{~V}$. The thickness was controlled by monitoring the charge passed, typically $250 \mu \mathrm{C}$. These charges were calibrated by measuring the thickness using a profilometer (Bruker Dektak XT). The resulting PANI-coated electrodes were blue-green in color. Electrodes were then rinsed with methanol and water, to remove unreacted monomers and oligomers from the surface, and stored dry.

\subsection{Electrochemical Growth of Gold Nanostructures}

Gold was deposited on the PANI-coated electrodes from $5 \mathrm{mmol} / \mathrm{L}, 25 \mathrm{mmol} / \mathrm{L}$ or $50 \mathrm{mmol} / \mathrm{L}$ $\mathrm{KAuCl}_{4}$ in $2 \mathrm{~mol} / \mathrm{L} \mathrm{HBF}_{4}$. The potential pulse routine was as follows (all potentials are versus $\mathrm{Ag} / \mathrm{AgCl}$ in $0.1 \mathrm{~mol} / \mathrm{L} \mathrm{KCl}$ ): $-200 \mathrm{mV}$ held for $6 \mathrm{~s}$, then $400 \mathrm{mV}$ held for 6 seconds, and repeat 100 times for a total deposition time of $1200 \mathrm{~s}$. The films were then thoroughly rinsed with water and cycled in $2 \mathrm{~mol} / \mathrm{L} \mathrm{HBF}_{4}$ followed by $2.5 \mathrm{mmol} / \mathrm{L}$ potassium ferricyanide in $0.1 \mathrm{~mol} / \mathrm{L} \mathrm{KCl}$ to confirm the retention of electrochemical activity after gold deposition.

\subsection{Scanning Electron Microscopy}


Scanning electron microscopy (SEM) was carried out with an Ultra-60 FESEM (Zeiss). The secondary emission detector was used with a working distance of approximately $5 \mathrm{~mm}$ and an accelerating voltage of $2.00 \mathrm{kV}$.

\subsection{SERS Measurements}

Raman spectra were acquired using a Renishaw S1000 micro-Raman spectrometer (Renishaw, Gloucestershire, UK) consisting of a Leica DMLM microscope coupled to a $250 \mathrm{~mm}$ focal length imaging spectrograph with a proprietary deep depletion, thermoelectrically cooled $\left(-70{ }^{\circ} \mathrm{C}\right)$ charge-coupled device. All spectra were taken with a $633 \mathrm{~nm} \mathrm{HeNe}$ laser. The total power at the sample was $1.1 \mathrm{~mW}$ (unless otherwise noted), the total integration time was $1.0 \mathrm{~s}$, and a 10x objective was used. To obtain each resultant spectrum, 81 spectra from 81 different spots were averaged to determine the spot-to-spot uniformity. The spots were taken from a $200 \mu \mathrm{m}$ by 200 $\mu \mathrm{m}$ square in the center of the electrode. Within this square, spectra were taken at spatial steps of $25 \mu \mathrm{m}$. An automated x-y stage was used to accomplish this sampling procedure. The background of each spectrum was fit using a $7^{\text {th }}$ order polynomial and subtracted. The baseline-subtracted spectra were averaged and smoothed using a moving average with a window of 5 points. Each set of 81 measurements was performed on four identically fabricated electrodes to determine electrode-to-electrode reproducibility.

For the SERS measurements, $50 \mu \mathrm{L}$ of R6G solution in water, diluted to various concentrations, was drop-cast on the electrode array and allowed to dry in a fume hood for at least 3 hours. The covered surface area of the $50 \mu \mathrm{L} \mathrm{R} 6 \mathrm{G}$ drop varied but was approximately $200 \mathrm{~mm}^{2}$. Using the drop volume $(50 \mu \mathrm{L})$, drop area $\left(200 \mathrm{~mm}^{2}\right)$, the $\mathrm{R} 6 \mathrm{G}$ concentration $(10 \mathrm{nmol} / \mathrm{L}$ to 1 $\mathrm{mmol} / \mathrm{L})$, and the laser spot size $(5 \mu \mathrm{m})$, the approximate number of R6G molecules measured in each spectrum could be calculated. For instance, a concentration of $100 \mu \mathrm{mol} / \mathrm{L}$ R6G corresponds to approximately $3 \times 10^{8}$ molecules in the laser spot.

\section{Results and Discussion}

\subsection{Electrochemical Deposition}

For this work, a two-step electrochemical process was used to fabricate nanostructured PANIAu composite materials on electrode arrays. In this procedure, electrochemically-grown PANI was used as a template for the binding, nucleation and electrochemical growth of gold nanostructures. PANI films were grown at a constant potential of $900 \mathrm{mV}$ from $0.1 \mathrm{~mol} / \mathrm{L}$ aniline in $2 \mathrm{~mol} / \mathrm{L} \mathrm{HBF}_{4}$ aqueous solution, as shown in Figure 1. The dotted line (right axis) in Figure 1 shows the charge passed. Deposition was limited to $250 \mu \mathrm{C}\left(31.8 \mathrm{mC} / \mathrm{cm}^{2}\right)$, resulting in film thicknesses of $\sim 50 \mathrm{~nm}$. Electrodeposited PANI films of this thickness are dense, smooth and provide a uniform surface for the growth of gold. While chloroaurate can also be spontaneously reduced on thicker films, the resulting PANI-Au composites were found not to be optimal for SERS measurements because only isolated, widely dispersed gold nanoparticles were formed. For these reasons, only gold nanostructures grown on thin PANI films were investigated in this work.

Deposition of gold on these thin PANI films was carried out under chronoamperometric control as shown in Figure 2. The potential was repeatedly stepped between $-200 \mathrm{mV}$ and $400 \mathrm{mV}$ with a hold time at each step of 6 seconds. Potential steps between these two values switch the redox state of the PANI film, a process determined to be necessary for the growth of SERS-active and electrochemically-active PANI-Au nanostructures. This repeated switching of the conducting state of PANI induces a change in the location of the active electrochemical interface.[29] Specifically, upon stepping from $-200 \mathrm{mV}$ to $400 \mathrm{mV}$ the PANI film undergoes the leucoemeraldine to emeraldine transition. This transition is accompanied by an egress of protons to maintain charge neutrality and an increase in the conductivity of the PANI film.[30] Note that chloroaurate is reduced at both potentials, as evidenced by the cathodic current during each step. 
At $400 \mathrm{mV}$, reduction of chloroaurate occurs at the PANI-electrolyte interface and the resulting metallic gold is deposited on the polymer chains as discrete nanoparticles. On the other hand, at $200 \mathrm{mV}$ PANI is only slightly conducting and the electrochemical interface is shifted laterally to the Pt electrode where the reduction of chloroaurate takes place. As this process is repeated, in this work 100 times, the gold nanoparticles along the polymer chains merge with the gold film growing underneath, forming an electrical contact between the gold nanoparticles and the Pt electrode. This contact is necessary for insuring the electrochemical activity of the PANI-Au composite at neutral $\mathrm{pH}$, as will be shown below. We also suspect that the repeated protonation and deprotonation of the nitrogens in the PANI chains plays a role in the resulting nanostructure of the gold film, as these nitrogens are also the binding sites for the chloroaurate anions. Further research is needed to clarify this mechanism.

In order to tailor the nanostructure of the PANI-Au electrodes, the deposition parameters were systematically varied across the electrode array, including the background electrolyte (i.e. identity of the acid), the $\mathrm{pH}$, and the chloroaurate concentration. Of these, the identity of the acid and the chloroaurate concentration were found to have the largest effect. The role of the identity of the acid will be examined in a later study. In this paper we focus on comparing PANI-Au films where the only variable examined is the concentration of chloroaurate used during deposition. Figure 3 shows SEM images for depositions from three different concentrations of chloroaurate: $5 \mathrm{mmol} / \mathrm{L}$, $25 \mathrm{mmol} / \mathrm{L}$ and $50 \mathrm{mmol} / \mathrm{L}$. The $5 \mathrm{mmol} / \mathrm{L}$ deposition results in arrays of somewhat spherical gold nanoparticles. These particles are approximately $250 \mathrm{~nm}$ in diameter and have an interparticle spacing of approximately $5 \mathrm{~nm}$ to $50 \mathrm{~nm}$. This range of interparticle spacing is conducive to the formation of SERS hot-spots.[31] On the other hand, the morphologies obtained from $25 \mathrm{mmol} / \mathrm{L}$ and $50 \mathrm{mmol} / \mathrm{L} \mathrm{KAuCl}_{4}$ are nanodendritic and nanosheet-like, respectively. These nanostructures have many sharp edges and points, which can act as antennae for SERS enhancement.[32] For the rest of this paper, the films deposited from $5 \mathrm{mmol} / \mathrm{L}, 25 \mathrm{mmol} / \mathrm{L}$ and $50 \mathrm{mmol} / \mathrm{L} \mathrm{KAuCl}_{4}$ will be referred to as nanoparticle, nanodendrite and nanosheet films, respectively.

The resulting nanostructure of the gold films was found to be relatively insensitive to small changes in the two potentials used, as long as the potential steps result in the movement of the deposition interface, i.e. a leucoemeraldine-emeraldine transition is necessary. Likewise, resulting gold nanostructures were found to be insensitive to small variations in the time spent at each step. But if the step time was very long, approaching a constant potential deposition, smoother gold films were obtained. Or, if the hold time was too short the kinetics of the PANI oxidation/reduction become sluggish compared with the step rate resulting, again, in smoother gold films. Hold times of $\sim 3-10$ seconds was found to be satisfactory for attaining the nanostructured interfaces.

\subsection{Electrochemical Characterization of PANI-Au Electrodes}

Next, the electrochemical activities of the PANI-Au electrodes were investigated. First, the PANI electrodes were cycled in aqueous $2 \mathrm{~mol} / \mathrm{L} \mathrm{HBF}_{4}$ before gold deposition (Figure 4a, solid line). In acidic solutions, PANI is conducting and two well-characterized redox waves are observed. The first redox wave at approximately $0.1 \mathrm{~V}$ is the leucoemeraldine-to-emeraldine transition, which is associated with the egress/ingress of protons.[30] The second redox wave at around $0.75 \mathrm{~V}$ is the emeraldine to pernigraniline transition and is accompanied by the ingress/egress of $\mathrm{BF}_{4}{ }^{-}$anions. The smaller redox process in the middle of these two, around $0.5 \mathrm{~V}$, is typically attributed to the reduction and release of degradation products formed during deposition.[30] After gold deposition, the electrodes were cycled again in $2 \mathrm{~mol} / \mathrm{L} \mathrm{HBF}_{4}$ to determine if PANI retains its electrochemical activity after the deposition of Au. As seen in 4a, the characteristic redox processes of PANI are present, but they are altered slightly in all three cases of deposited gold. In particular, the middle peak, corresponding to the reduction of the 
degradation products, appears to increase and there is an additional peak around $0.4 \mathrm{~V}$ that is more prevalent in the nanodendrite and nanosheet films. The increase of the middle peak at $0.5 \mathrm{~V}$ suggests that the reduction and subsequent deposition of gold causes additional oxidative damage to the PANI films, without loss of electrochemical activity. This damage is to be expected since the PANI oxidation is concurrent with chloroaurate reduction. The additional peak, seen as a shoulder around $0.4 \mathrm{~V}$, may be associated with the reduction of unreacted chloroaurate. Along with these slight changes in the redox behavior of the PANI-Au films, the capacitances of the films also appear to increase as the chloroaurate concentration is increased. This suggests that the gold layer partially blocks the ingress and egress of $\mathrm{BF}_{4}{ }^{-}$anions and protons, effectively trapping some of these charges in the PANI film.

For potential biosensing applications, the EC behavior in physiological $\mathrm{pH}$ must be preserved. To assess this capability the electrodes were cycled in $2.5 \mathrm{mmol} / \mathrm{L}$ potassium ferricyanide in 0.1 $\mathrm{mol} / \mathrm{L} \mathrm{KCl}$ (Figure $4 \mathrm{~b}$ ). Under these conditions the redox wave of potassium ferricyanide is clearly present. Both the redox and capacitive currents increase with the amount of gold deposited. The increase in capacitive current was observed already and again may be attributed to partial trapping of ions within the PANI-Au film. The redox activity of these films in neutral $\mathrm{pH}$ is somewhat surprising considering that PANI is only slightly conducting under these conditions. The PANI-Au films were carefully examined under both optical microscope and SEM for pinholes that may have formed during or after deposition, but none were found. The electrochemical activity of the PANI-Au electrodes at neutral $\mathrm{pH}$ reinforces the view that these films are not discrete bilayers with PANI at the bottom and gold on top. Instead, some of the chloroaurate penetrates and is reduced within the PANI film and on top of the Pt electrode, particularly at a deposition potential of $-200 \mathrm{mV}$. This penetration of chloroaurate during deposition allows the resulting metallic gold to make electrical contact directly with the $\mathrm{Pt}$ electrode and is crucial for the electroactivity in $\mathrm{pH}$ ranges relevant for biosensing. As a result, in acidic solution the electrode possesses the properties of the PANI film, as evidenced by its characteristic redox behavior, but at neutral $\mathrm{pH}$ when the PANI is inactive, the interpenetrating gold acts as an electrical shunt maintaining a conducting Au electrode surface.

As the amount of gold deposited increases, the electroactive area also increases due to the increased nanoscale roughness (see inset in Figure 4b). The inset shows the electrode area, A, for each electrode type, calculated from the following equation:

$$
I_{p, c}=-2.69 \times 10^{5} n^{3 / 2} A D_{O}^{1 / 2}[O]_{\infty} v^{1 / 2}
$$

where $I_{p, c}$ is the reduction peak potential, $n$ is the number of electrons transferred, $D_{O}$ is the diffusion coefficient of the oxidized species, $[O]_{\infty}$ is the concentration of oxidized species in the bulk of the solution and $v$ is the scan rate. The diffusion coefficient of ferricyanide in $0.1 \mathrm{M} \mathrm{KCl}$ is $0.72 \times 10^{-5} \mathrm{~cm}^{2} \mathrm{sec}^{-1}$.[33] The area increases as the concentration of chloroaurate used during deposition is increased. Because the footprint of the electrodes remains constant, this indicates that the roughness factor increases, as would be expected from examination of the electron micrographs in Figure 3.

\subsection{SERS Performance of PANI-Au Electrodes}

Figure 5a shows the Raman spectra of $100 \mu \mathrm{mol} / \mathrm{L}$ R6G drop-cast on each of the electrode types (see Experimental section for details). This concentration of R6G corresponds to approximately $3 \times 10^{8}$ molecules in the laser spot. For comparison, $1 \mathrm{mmol} / \mathrm{L} \mathrm{R6G}$ drop-cast on a smooth gold reference surface (non-enhancing) is shown as a solid line. Note that the laser power for this reference spectrum is 12 times greater than used to acquire the various SERS spectra on PANI-Au. Figure 5b shows the average peak intensity at $1362 \mathrm{~cm}^{-1}$ for the same coverage of R6G with error bars representing \pm one standard deviation. The average spot-to-spot relative standard 
deviations across the films are $8 \%, 35 \%$ and $60 \%$ for the nanoparticle, nanodendritic and nanosheet films, respectively. The electrode-to-electrode relative standard deviations are $12 \%$, $32 \%$ and $25 \%$. These spot-to-spot and electrode-to-electrode relative standard deviations for the nanoparticle electrodes are adequate for reproducible EC-SERS applications and, under these conditions, comparable to commercially available SERS-only substrates, which typically guarantee spot-to-spot relative standard deviations of around 5\% or more.

From Figure 5, the nanoparticle PANI-Au films not only have the best uniformity and reproducibility, but also have the highest EF, approximately 3 times greater than the nanodendrites and nanosheets. Differences in the localized surface plasmon resonances at $633 \mathrm{~nm}$ between the three electrode types may be responsible for the differences in EFs: it is possible that the nandendritic and nanosheet films may perform better at a different excitation wavelength. The response curve of the nanoparticle electrodes to increasing surface coverage of R6G is shown in Figure 6 (left axis). The intensity of the R6G peak at $1362 \mathrm{~cm}^{-1}$ increases logarithmically with increasing concentration. From the average intensities at each concentration, the SERS substrate EFs (SSEF) were calculated using the following equation:

$$
S S E F=\frac{I_{\text {SERS }} / N_{\text {surf }}}{I_{\text {ref }} / N_{\text {ref }}}
$$

where $I_{\text {SERS }}$ is the measured SERS intensity, $N_{\text {surf }}$ is the number of molecules measured on the SERS surface, $I_{\text {ref }}$ is the non-SERS Raman intensity (of the same molecule and vibrational mode) and $N_{\text {ref }}$ is the number of molecules measured on the reference surface.[34] SSEFs can depend on a number of factors, including surface coverage, analyte molecule, and Raman cross-section.[35]

The SSEFs increase from $1.9 \times 10^{3}$ at $100 \mu \mathrm{mol} / \mathrm{L}$ to $1.1 \times 10^{6}$ at $10 \mathrm{nmol} / \mathrm{L}$. This apparent increase in SSEF with decreasing surface coverage is a consequence of the localized nature of the enhanced electromagnetic field at the surface of the hot-spots, which extends only about $5 \mathrm{~nm}$ from the surface.[36] Assuming the upper limit for the surface coverage of a single R6G molecule to be $222 \AA^{2}$, [37] and using the electrode surface area calculated from Figure $4 \mathrm{~b}$, a $50 \mu \mathrm{L}$ drop of $100 \mu \mathrm{mol} / \mathrm{L}$ R6G on the nanoparticle films corresponds to roughly 34 layers of R6G on the nanoparticle electrode surface. Using a lower limit for stacking distances of $3.5 \AA$, corresponding to typical stacking distances found in $\pi-\pi$ systems, and in particular the calculated stacking distance of R6G dimers, [38] the minimum R6G thickness for 34 layers is approximately $12 \mathrm{~nm}$. While this thickness is only approximate, it does serve to highlight an important feature of SERS measurements: at supermonolayer coverages like these, molecules stacked beyond the spatial extent of the hot-spots contribute negligibly to the overall signal. This results in a saturation of the response and a decrease of the calculated SSEF.

Better SSEF estimates can be obtained from lower concentrations, which avoid saturating hotspots (for instance, a concentration of $10 \mathrm{nmol} / \mathrm{L}$ corresponds to only $\sim 0.0034$ layers of R6G), unfortunately the spot-to-spot uniformity suffers. At $100 \mu \mathrm{mol} / \mathrm{L}$ R6G, the relative standard deviations are small, around $8 \%$. As the R6G concentration is reduced to $10 \mathrm{nmol} / \mathrm{L}$, the occupancy of hot-spots becomes spatially inhomogeneous, resulting in large relative standard deviations up to $67 \%$. These same trends were also observed when depositing increasing amounts of R6G onto commercially available Klarite substrates. On these well-ordered, lithographically fabricated substrates, the spot-to-spot relative standard deviation decreased from $16 \%$ at 10 $\mathrm{nmol} / \mathrm{L}$ to $3.9 \%$ at $0.1 \mathrm{mmol} / \mathrm{L}$ while the SSEF decreased from $5.4 \times 10^{6}$ at $10 \mathrm{nmol} / \mathrm{L}$ to $8.0 \times 10^{3}$ at $100 \mu \mathrm{mol} / \mathrm{L}$. Recently Darby et al. have explored the distribution of analyte molecules on nanoparticles and the consequences for the uniformity of the SERS signal.[39] The competition between diffusion of the analyte and adsorption on the surface, which takes place during solvent evaporation, can lead to inhomogeneity in surface coverage. At higher concentrations, where supermonolayer coverages are obtained, this inhomogeneity tends to average out. 
The bimodal biosensing applications we envision for these electrodes involve the deposition of surface-bound monolayers or sub-monolayers of thiol-labeled moieties to facilitate selective binding of target molecules, e.g. single-stranded DNA. Thus, the SERS performance of these materials with monolayer coverage of target molecules provides a better metric for biosensing applications. In order to guarantee uniform monolayer coverage, the PANI-Au nanoparticle films were immersed in $10 \mathrm{mmol} / \mathrm{L} 4-\mathrm{MBA}$ in ethanol for 24 hours. Using the peak at $1080 \mathrm{~cm}^{-1}$, the SSEF was found to be $\sim 1.5 \times 10^{5}$ with a spot-to-spot relative standard deviation of $\sim 21 \%$. Interestingly, this SSEF is less than the maximum found for submonolayer coverage using R6G, which was up to $1.1 \times 10^{6}$, which may be contributed to the resonance condition of R6G at 633 $\mathrm{nm}$.[35] Additionally, it is possible that the R6G molecules may preferentially deposit in the spaces between the nanoparticles, where the enhancement is predicted to be highest. Such preferential deposition of 1,3,5-trinitroperhydro-1,3,5-triazine within pyramidal wells has recently been reported.[40] Regardless, we would expect enhancements in the range of $10^{5}$ to $10^{6}$, an acceptable level for the intended applications we envision.

\section{Conclusions}

SERS-active PANI-Au electrodes were fabricated using electrochemically deposited PANI as a template for the electrochemical reduction of electrostatically-bound chloroaurate. In addition to its role in providing binding sites for chloroaurate, the PANI film was used to laterally shift the location of the electrochemical interface, and therefore the location of gold deposition. The deposition technique described in this paper builds upon the work of Janata et al. but differs in two key ways. Our method does not require the dissolution of gold from the underlying working electrode; instead the chloroaurate in the $\mathrm{HBF}_{4}$ solution allows control over the concentration of gold, providing the ability to tune the gold nanostructures by changing the chloroaurate concentration. Secondly, while Janata et al. primarily utilize chronopotentiometric techniques to produce gold-polyaniline composites, they also explore potentiostatic preparation similar to the technique outline here. The main difference is in the potentials used: here we step between the non-conducting leucoemeraldine and conducting emeraldine forms of PANI. The negative potential required to completely reduce the PANI film provides a stronger driving force for the deposition of dense nanostructured gold.

This technique results in tunable gold nanostructures within and on top of the PANI films. Most importantly, the interpenetrated PANI-Au films are electrochemically active in both neutral and acidic solutions. Changing the concentration of chloroaurate used during deposition produces drastic changes to the resulting nanostructures of the deposited gold, i.e. nanoparticles, nanodendrites, and nanosheets can be obtained. The approximate SSEFs, spot-to-spot uniformity, and electrode-to-electrode reproducibility were investigated using R6G and 4-MBA as SERS indicator molecules. Gold nanostructures deposited from $5 \mathrm{mmol} / \mathrm{L}$ chloroaurate, with nanoparticle morphology, performed the best with an average SSEF as high as $1.1 \times 10^{6}$ using R6G and $1.5 \times 10^{5}$ using monolayer coverage of 4-MBA It should be noted that this technique could be applied to a number of conducting polymers able to bind metal salts, including polypyrrole and polythiophine. The unique potential- and $\mathrm{pH}$-dependent electrochemical behavior of such interpenetrating polymer-metal films are yet to be explored and may have applications in chemical and biological sensing, energy storage, and organic electronics.

\section{Acknowledgements}

The authors thank Christopher Montgomery and Michael Carrier for advice and assistance during the fabrication of the electrode array. The authors also thank Aaron Urbas for assistance with the SERS measurements. Research was performed in part at the NIST Center for Nanoscale Science and Technology. R. M. W. was supported by a NIST Postdoctoral Research Associateship Award administered through the National Research Council. 


\section{Notes}

$\dagger$ Certain commercial equipment, instruments, or materials are identified in this document. Such identification is not intended to imply recommendation or endorsement by the National Institute of Standards and Technology, nor intended to imply that the products identified are necessarily the best available for the purpose.

\section{References}

[1] A. Hierlemann, R. Gutierrez-Osuna, Higher-order chemical sensing, Chem. Rev., 108 (2008) 563-613.

[2] E. Cortes, P.G. Etchegoin, E.C. Le Ru, A. Fainstein, M.E. Vela, R.C. Salvarezza, Electrochemical Modulation for Signal Discrimination in Surface Enhanced Raman Scattering (SERS), Anal. Chem., 82 (2010) 6919-6925.

[3] Z.Q. Tian, B. Ren, Adsorption and reaction at electrochemical interfaces as probed by surface-enhanced Raman spectroscopy, Annu. Rev. Phys. Chem., 55 (2004) 197-229.

[4] A. Krolikowska, Surface-enhanced resonance Raman scattering (SERRS) as a tool for the studies of electron transfer proteins attached to biomimetic surfaces: Case of cytochrome c, Electrochim. Acta, 111 (2013) 952-995.

[5] D.Y. Wu, J.F. Li, B. Ren, Z.Q. Tian, Electrochemical surface-enhanced Raman spectroscopy of nanostructures, Chem. Soc. Rev., 37 (2008) 1025-1041.

[6] A. Campion, P. Kambhampati, Surface-enhanced Raman scattering, Chem. Soc. Rev., 27 (1998) 241-250.

[7] P.G. Etchegoin, E.C. Le Ru, Basic Electromagnetic Theory of SERS, in: Surface Enhanced Raman Spectroscopy, Wiley-VCH Verlag GmbH \& Co. KGaA, 2010, pp. 1-37.

[8] H.X. Xu, E.J. Bjerneld, M. Kall, L. Borjesson, Spectroscopy of single hemoglobin molecules by surface enhanced Raman scattering, Phys. Rev. Lett., 83 (1999) 4357-4360.

[9] S. McAughtrie, K. Faulds, D. Graham, Surface enhanced Raman spectroscopy (SERS): Potential applications for disease detection and treatment, J. Photochem. Photobiol. C-Photochem. Rev., 21 (2014) 40-53.

[10] P. Xu, X.J. Han, B. Zhang, Y.C. Du, H.L. Wang, Multifunctional polymer-metal nanocomposites via direct chemical reduction by conjugated polymers, Chem. Soc. Rev., 43 (2014) 1349-1360.

[11] G. Ciric-Marjanovic, Recent advances in polyaniline composites with metals, metalloids and nonmetals, Synth. Met., 170 (2013) 31 56.

[12] R.J. Tseng, J.X. Huang, J. Ouyang, R.B. Kaner, Y. Yang, Polyaniline nanofiber/gold nanoparticle nonvolatile memory, Nano Lett., 5 (2005) 1077-1080.

[13] S. Liu, H.M. Xu, J.F. Ou, Z.P. Li, S.R. Yang, J.Q. Wang, A feasible approach to the fabrication of gold/polyaniline nanofiber composites and its application as electrocatalyst for oxygen reduction, Mater. Chem. Phys., 132 (2012) 500-504.

[14] C.O. Baker, B. Shedd, R.J. Tseng, A.A. Martinez-Morales, C.S. Ozkan, M. Ozkan, Y. Yang, R.B. Kanert, Size Control of Gold Nanoparticles Grown on Polyaniline Nanofibers for Bistable Memory Devices, ACS Nano, 5 (2011) 3469-3474.

[15] G. Majumdar, M. Goswami, T.K. Sarma, A. Paul, A. Chattopadhyay, Au nanoparticles and polyaniline coated resin beads for simultaneous catalytic oxidation of glucose and colorimetric detection of the product, Langmuir, 21 (2005) 1663-1667.

[16] A. Dey, A. Kaushik, S.K. Arya, S. Bhansali, Mediator free highly sensitive polyaniline-gold hybrid nanocomposite based immunosensor for prostate-specific antigen (PSA) detection, J. Mater. Chem., 22 (2012) 14763-14772.

[17] M. Srivastava, S.K. Srivastava, N.R. Nirala, R. Prakash, A chitosan-based polyaniline-Au nanocomposite biosensor for determination of cholesterol, Anal. Methods, 6 (2014) 817-824.

[18] K. Mallick, M.J. Witcomb, M.S. Scurrell, Gold in polyaniline: Recent trends, Gold Bull., 39 (2006) 166-174.

[19] Y.Y. Li, H.J. Schluesener, S.Q. Xu, Gold nanoparticle-based biosensors, Gold Bull., 43 (2010) 29-41.

[20] J.M. Pingarron, P. Yanez-Sedeno, A. Gonzalez-Cortes, Gold nanoparticle-based electrochemical biosensors, Electrochim. Acta, 53 (2008) 5848-5866.

[21] Y.Y. Xia, T.J. Li, J. Chen, Compact large area and high-quality Au film with a hierarchical structure and its application in SERS, Phys. Chem. Chem. Phys., 15 (2013) 11900-11903.

[22] S.W. Li, P. Xu, Z.Q. Ren, B. Zhang, Y.C. Du, X.J. Han, N.H. Mack, H.L. Wang, Fabrication of Thorny Au Nanostructures on Polyaniline Surfaces for Sensitive Surface-Enhanced Raman Spectroscopy, ACS Appl. Mater. Interfaces, 5 (2013) 49-54.

[23] T.K. Sarma, A. Chattopadhyay, One pot synthesis of nanoparticles of aqueous colloidal polyaniline and its Au-nanoparticle composite from monomer vapor, J. Phys. Chem. A, 108 (2004) 7837-7842.

[24] K. Huang, Y.J. Zhang, Y.Z. Long, J.H. Yuan, D.X. Han, Z.J. Wang, L. Niu, Z. Chen, Preparation of highly conductive, selfassembled gold/polyaniline nanocables and polyaniline nanotubes, Chem.-Eur. J., 12 (2006) 5314-5319.

[25] A.P. Jonke, M. Josowicz, J. Janata, Polyaniline Doped with Atomic Gold, J. Electrochem. Soc., 158 (2011) E147-E151.

[26] H.F. Zhang, R.X. Liu, J.B. Zheng, Seed-mediated synthesis of polyaniline/Au nanocomposite and its application for a cholesterol biosensor, Synth. Met., 167 (2013) 5-9.

[27] D.W. Hatchett, M. Josowicz, J. Janata, D.R. Baer, Electrochemical formation of Au clusters in polyaniline, Chem. Mat., 11 (1999) 2989-2994.

[28] K. Qian, H.L. Liu, L.B. Yang, J.H. Liu, Designing and fabricating of surface-enhanced Raman scattering substrate with high density hot spots by polyaniline template-assisted self-assembly, Nanoscale, 4 (2012) 6449-6454.

[29] J.A. Smith, M. Josowicz, J. Janata, Gold-polyaniline composite - Part I. Moving electrochemical interface, Phys. Chem. Chem. Phys., 7 (2005) 3614-3618.

[30] A.P. Jonke, M. Josowicz, J. Janata, M.H. Engelhard, Electrochemically Controlled Atom by Atom Deposition of Gold to Poly aniline, J. Electrochem. Soc., 157 (2010) P83-P87.

[31] C.J. Choi, S. Semancik, Effect of interdome spacing on the resonance properties of plasmonic nanodome arrays for label-free optical sensing, Opt. Express, 21 (2013) 28304-28313. 
[32] Y.F. Chan, C.X. Zhang, Z.L. Wu, D.M. Zhao, W. Wang, H.J. Xu, X.M. Sun, Ag dendritic nanostructures as ultrastable substrates for surface-enhanced Raman scattering, Appl. Phys. Lett., 102 (2013) 5.

[33] S.J. Konopka, B. McDuffie, DIFFUSION COEFFICIENTS OF FERRICYANIDE AND FERROCYANIDE IONS IN AQUEOUS MEDIA, USING TWIN-ELECTRODE THIN-LAYER ELECTROCHEMISTRY, Anal. Chem., 42 (1970) 1741-\&.

[34] C.J. Choi, Z.D. Xu, H.Y. Wu, G.L. Liu, B.T. Cunningham, Surface-enhanced Raman nanodomes, Nanotechnology, 21 (2010) 7.

[35] E.C. Le Ru, E. Blackie, M. Meyer, P.G. Etchegoin, Surface enhanced Raman scattering enhancement factors: a comprehensive study, J. Phys. Chem. C, 111 (2007) 13794-13803.

[36] N.E. Marotta, K.R. Beavers, L.A. Bottomley, Limitations of Surface Enhanced Raman Scattering in Sensing DNA Hybridization Demonstrated by Label-Free DNA Oligos as Molecular Rulers of Distance-Dependent Enhancement, Anal. Chem., 85 (2013) 1440-1446.

[37] R. Gupta, W.A. Weimer, High enhancement factor gold films for surface enhanced Raman spectroscopy, Chem. Phys. Lett., 374 (2003) 302-306.

[38] J. Zhao, L. Jensen, J. Sung, S. Zou, G.C. Schatz, R.P. Van Duyne, Interaction of Plasmon and MolecularResonances for Rhodamine 6G Adsorbed on Silver Nanoparticles, J. Am. Chem. Soc., 129 (2007) 7647-7656.

[39] B.L. Darby, E.C. Le Ru, Competition between Molecular Adsorption and Diffusion: Dramatic Consequences for SERS in Colloidal Solutions, J. Am. Chem. Soc., 136 (2014) 10965-10973.

[40] S. Almaviva, S. Botti, L. Cantarini, R. Fantoni, S. Lecci, A. Palucci, A. Puiu, A. Rufoloni, Ultrasensitive RDX detection with commercial SERS substrates, J. Raman Spectrosc., 45 (2014) 41-46. 


\section{Figures and Captions}

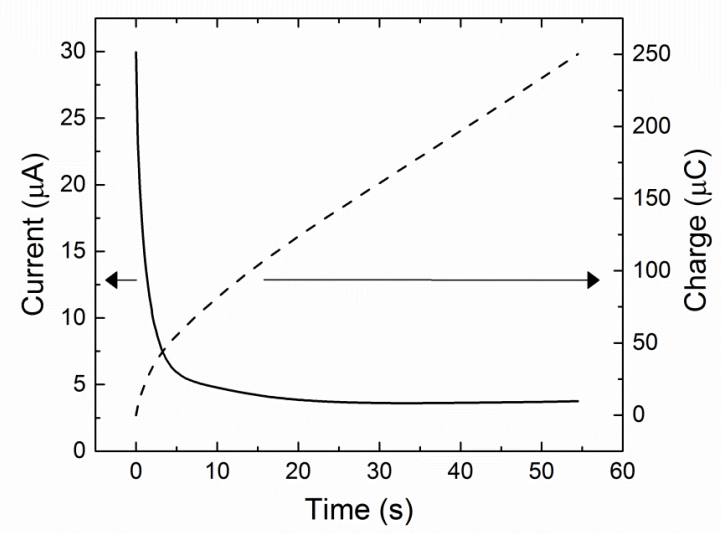

Figure 1 Potentiostatic deposition of polyaniline on a Pt electrode from $0.1 \mathrm{~mol} / \mathrm{L}$ aniline in $2 \mathrm{~mol} / \mathrm{L}$ $\mathrm{HBF}_{4}$ at $0.9 \mathrm{~V}$ vs. $\mathrm{Ag} / \mathrm{AgCl}$ (in $1 \mathrm{~mol} / \mathrm{L} \mathrm{KCl}$ ). The charge passed during deposition (dashed line, right axis) was monitored and deposition was typically terminated when $250 \mu \mathrm{C}$ was reached, resulting in film thicknesses of approximately $50 \mathrm{~nm}$.

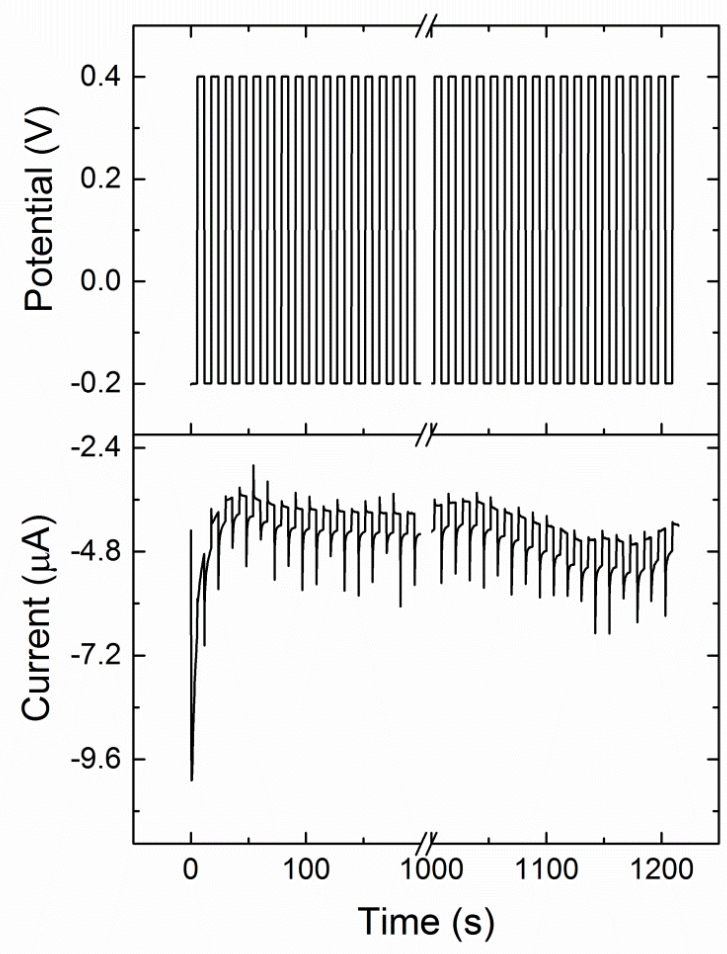

Figure 2 Reduction of $5 \mathrm{mmol} / \mathrm{L} \mathrm{KAuCl}_{4}$ in $2 \mathrm{~mol} / \mathrm{L} \mathrm{HBF}_{4}$ on a PANI-coated Pt electrode via potential pulses. The potential was stepped between $-0.2 \mathrm{~V}$ and $0.4 \mathrm{~V}$ with a hold time of 6 seconds at each potential for 100 total steps (see top). The current (bottom curve) is negative indicating that $\mathrm{KAuCl}_{4}$ is reduced at both potentials. 


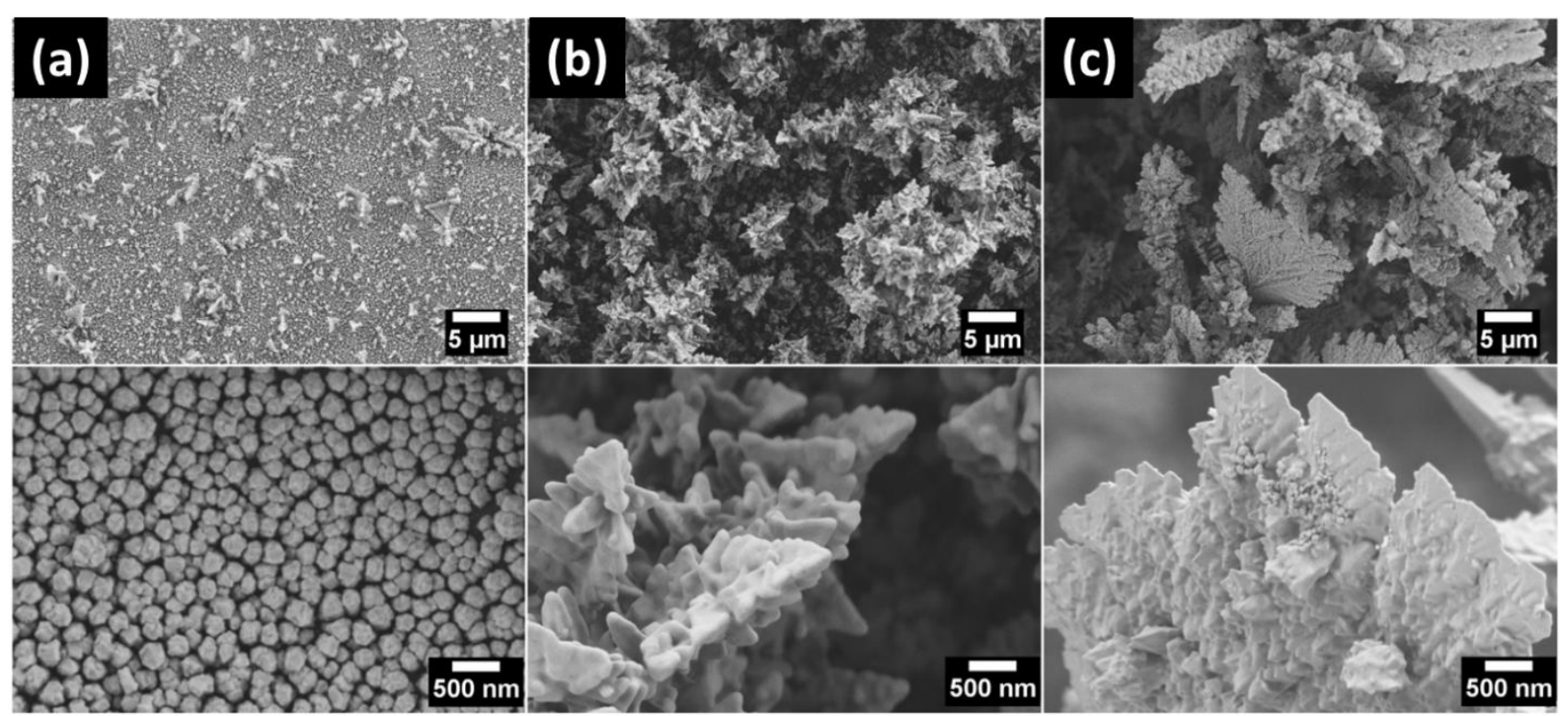

Figure 3 SEM images of three film morphologies deposited from three different $\mathrm{KAuCl}_{4}$ concentrations: (left column) nanoparticle structures deposited from $5 \mathrm{mmol} / \mathrm{L}$; (middle column) nanodendrites deposited from $25 \mathrm{mmol} / \mathrm{L}$; and (right column) nanosheets deposited from $50 \mathrm{mmol} / \mathrm{L}$.
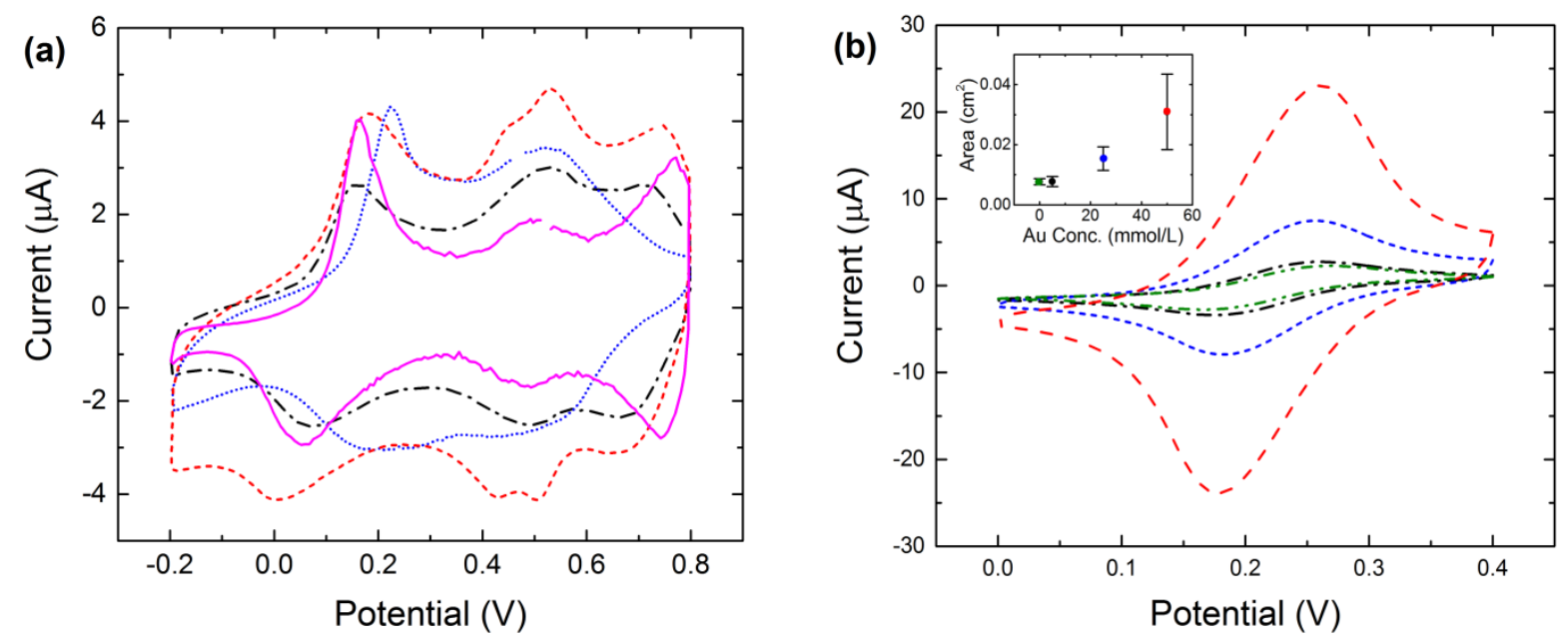

Figure 4 Electrochemical characterization of nanostructured PANI-Au electrodes, with gold deposited from $5 \mathrm{mmol} / \mathrm{L}, 25 \mathrm{mmol} / \mathrm{L}$ and $50 \mathrm{mmol} / \mathrm{L} \mathrm{KAuCl}_{4}$, shown in black (dash-dot), red (dash) and blue (dot), respectively. (a) Cyclic voltammograms of PANI and PANI-Au electrodes in $2 \mathrm{~mol} / \mathrm{L} \mathrm{HBF}_{4}$ between $-0.2 \mathrm{~V}$ and $0.8 \mathrm{~V}$ vs. $\mathrm{Ag} / \mathrm{AgCl}(1 \mathrm{~mol} / \mathrm{L} \mathrm{KCl})$ at a scan rate of $50 \mathrm{mV} / \mathrm{s}$. The $\mathrm{CV}$ of PANI alone is shown in purple (solid). (b) Cyclic voltammograms of three different PANI-Au films in $2.5 \mathrm{mmol} / \mathrm{L}$ $\mathrm{FeCN}$ in $0.1 \mathrm{~mol} / \mathrm{L} \mathrm{KCl}$ between $0.0 \mathrm{~V}$ and $0.4 \mathrm{~V}$ at a scan rate of $50 \mathrm{mV} / \mathrm{s}$. The $\mathrm{CV}$ on a bare Pt electrode is shown in green (dash-dot-dot). The electrode areas calculated from the peak heights are shown in the inset. 

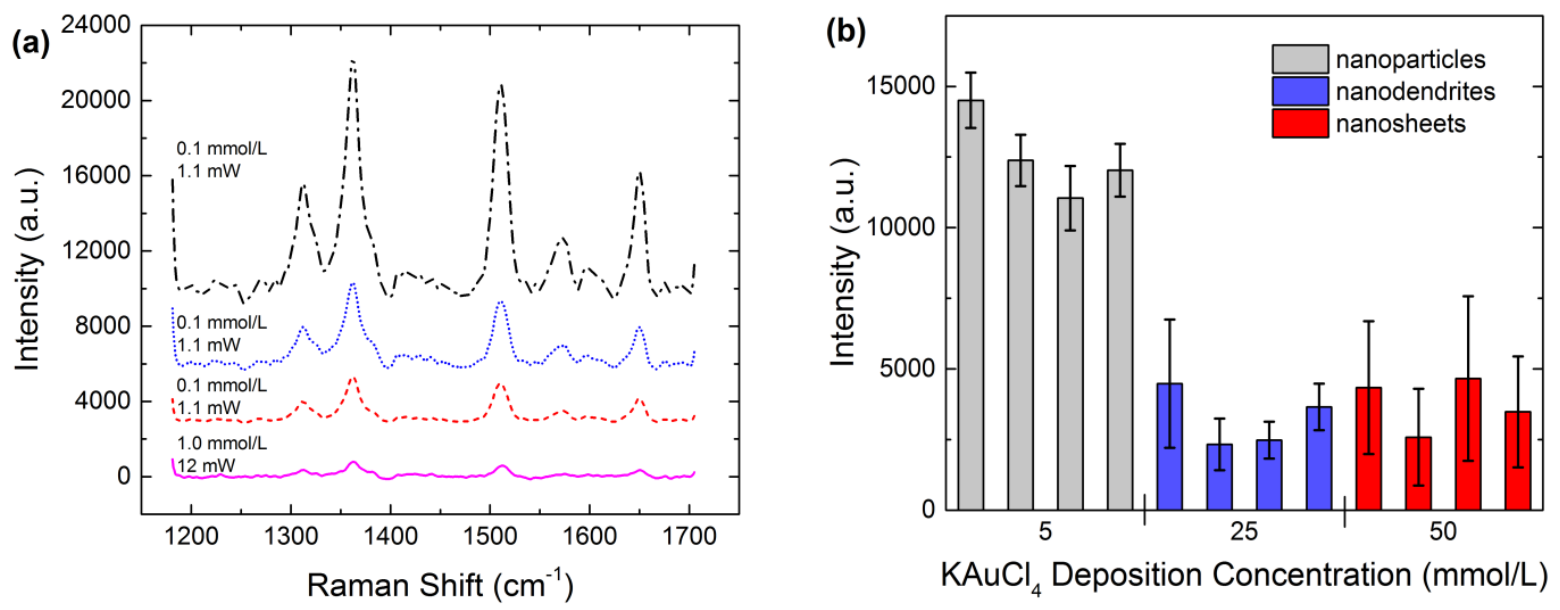

Figure 5 SERS characterization of nanostructured PANI-Au electrodes with gold deposited from 5 $\mathrm{mmol} / \mathrm{L}, 25 \mathrm{mmol} / \mathrm{L}$ and $50 \mathrm{mmol} / \mathrm{L} \mathrm{KAuCl}_{4}$, shown in black (dash-dot), red (dash), and blue (dot), respectively (same color coding as in Figure 4). (a) The spectra of $50 \mu \mathrm{L}$ of $0.1 \mathrm{mmol} / \mathrm{L}$ R6G (drop area is approximately $200 \mathrm{~mm}^{2}$ ) on the three different PANI-Au electrodes. Laser power is $1.1 \mathrm{~mW}$. Spectrum of $50 \mu \mathrm{L}$ of $1 \mathrm{mmol} / \mathrm{L} \mathrm{R6G}$ on smooth gold reference is shown in purple (solid) and was acquired at a laser power of $12 \mathrm{~mW}$. (b) SERS intensity at $1362.2 \mathrm{~cm}^{-1}$ of $50 \mu \mathrm{L}$ of $0.1 \mathrm{mmol} / \mathrm{L} \mathrm{R} 6 \mathrm{G}$ for four separate electrodes of each of the three different film morphologies. Error bars are \pm one standard deviation for 81 measurements collected across each electrode.

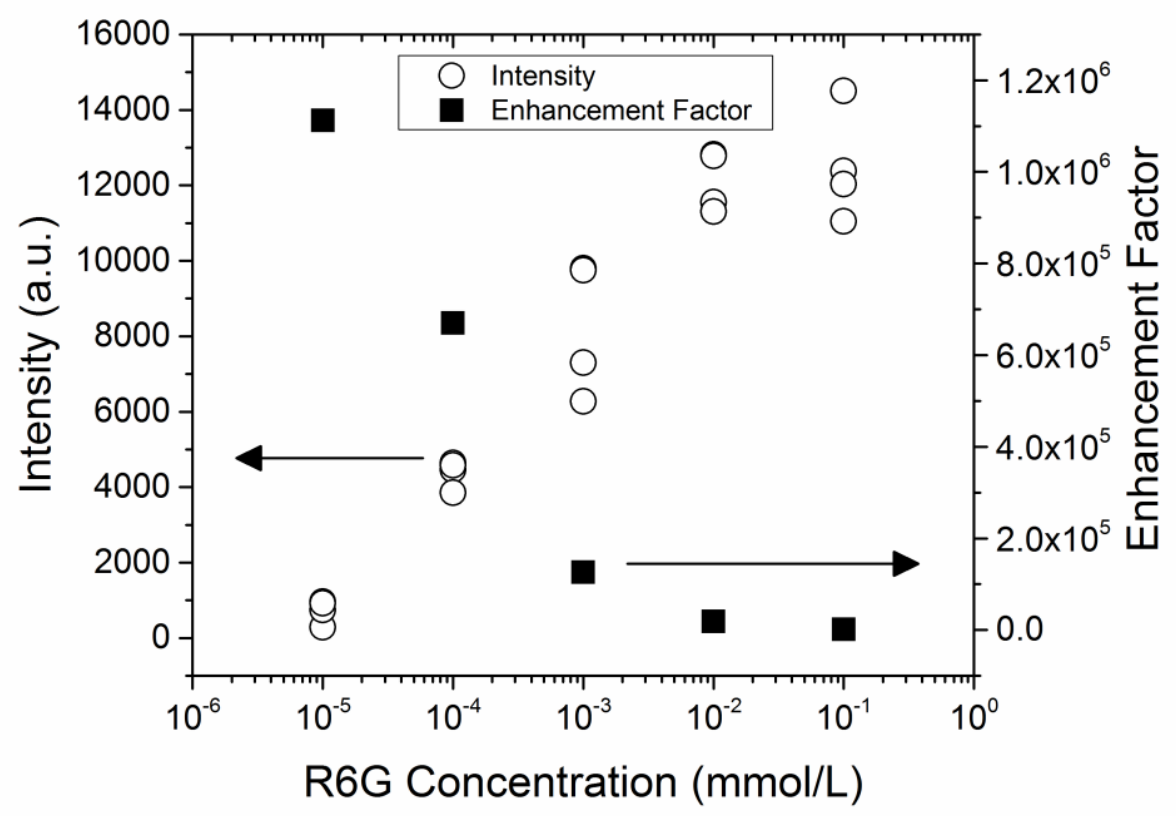

Figure 6 Concentration-dependent SERS response for the nanoparticle films shown in figure 5. The average intensities (at $1362.2 \mathrm{~cm}^{-1}$ ) for each concentration are plotted for the four identically prepared electrodes (hollow circles, left axis). The calculated average enhancement factors at each concentration are plotted as filled squares (right axis). The enhancement factors for the two highest concentrations, 0.1 $\mathrm{mmol} / \mathrm{L}$ and $0.01 \mathrm{mmol} / \mathrm{L}$, are $1.9 \times 10^{3}$ and $1.8 \times 10^{4}$ respectively. 